\title{
La equidad de género en la educación peruana
}

Gender equity in Peruvian education

Equidade de gênero na educação peruana

\author{
Elba Sissi Acevedo Rojas \\ eacevedor@uni.edu.pe \\ Universidad Nacional de Ingeniería - Perú \\ https://orcid.org/0000-0002-1090-6012
}

\section{RESUMEN}

Existe una agenda urgente como es la de incorporar en las políticas públicas nacionales el enfoque de género, con el objetivo de reducir o eliminar brechas de desigualdad entre hombres y mujeres en los diferentes ámbitos de desarrollo social, político, cultural, educativo; si bien el enfoque viene siendo discutido desde Jomtien en el año 1990, sin embargo, el Perú no cumplió con dicho acuerdo. En el año 2000 en Dakar, varios países incluido el Perú se sientan a la mesa y acuerdan incorporar la perspectiva de género en las políticas educativas, que se sustenta en el Objetivo de Desarrollo Sostenible (ODS 5); luego de aquel compromiso, la UNESCO realizó un diagnóstico respecto a la incorporación del acuerdo, que demostró que no se han cumplido en su totalidad con dicho compromiso. Además, de la falta de voluntad política se ha sumado una ideología religiosa que no acepta la incorporación de la perspectiva de género en la política educativa.

Palabras clave / Descriptores: Equidad de género en la educación. Género y educación. Derechos humanos en la educación. Igualdad de género.

\begin{abstract}
There is an urgent agenda such as incorporating the gender approach into national public policies, with the aim of reducing and/or eliminating inequality gaps between men and women in different areas of social, political, cultural, educational development, etc. This approach has been discussed since Jomtien in 1990; however, Peru did not comply with this agreement. In 2000 in Dakar, several countries, including Peru, sat at the table, and agreed to incorporate the gender perspective in educational policies, which is based on the Sustainable Development Goal (SDG 5), after that commitment, the UNESCO carried out a diagnosis regarding the incorporation of the agreement, which showed that this commitment has not been fully complied with. In addition, the lack of political will has added a religious ideology that does not accept the incorporation of the gender perspective in educational policy.
\end{abstract}

Keywords: Gender equity in education. Gender and education. Human rights in education. Gender equality.

\section{RESUMO}

Há uma agenda urgente como a de incorporar o enfoque de gênero nas políticas públicas nacionais, com o objetivo de reduzir ou eliminar as desigualdades entre homens e mulheres nas diferentes esferas do desenvolvimento social, político, cultural e educacional; embora a abordagem tenha sido discutida desde Jomtien em 1990, o Peru não cumpriu o referido acordo. No ano 2000, em Dakar, vários países, incluindo o Peru, sentam-se à mesa e concordam em incorporar a perspectiva de gênero nas políticas educacionais, que se baseia no Objetivo de Desenvolvimento Sustentável (ODS 5); após esse compromisso, a UNESCO fez um diagnóstico sobre a incorporação do acordo, que mostrou que o compromisso não foi totalmente cumprido. Além disso, a falta de vontade política acrescentou uma ideologia religiosa que não aceita a incorporação da perspectiva de gênero na política educacional.

Palavras chave: Equidade de gênero na educação. Gênero e educação. Direitos humanos na educação. Igualdade de gênero.

\section{INTRODUCCIÓN}

Este ensayo tuvo como objetivo determinar el avance de la implementación del enfoque de género en la política educativa. Este trabajo es de tipo teórico, exploratorio, no experimental y descriptivo que establece como pregunta de investigación: ¿Existe una educación inclusiva y con equidad de género en la política educativa peruana? Para la fase exploratoria se consideraron las siguientes partes en el cuerpo del trabajo: a) Género en la historia: derechos y ciudadanía, b) género 
y educación para cerrar brechas y c) políticas públicas para una educación con equidad. El primero buscó analizar la forma como se fue incorporando el concepto de género y también el de enfoque; no obstante, si bien las mujeres lo han ido incluyendo a los diferentes ámbitos de su vida que antes se consideraban ser exclusivos de hombres, aún falta mayores avances para lograr la igualdad necesaria. El segundo, las investigaciones revisadas han señalado que la educación es el elemento necesario por el cual se debe incorporar el enfoque de género, ya que en dicho sector aún se sigue perpetuando la violencia simbólica. Además, que es a través de los diferentes enfoques curriculares que se perpetúa la discriminación en contra de las mujeres, generando mayores brechas, incluso en ámbitos lejanos a la escuela. El tercero trata de las políticas públicas para una educación con equidad; al respecto, la investigación evidencia cómo las organizaciones internacionales acuerdan con los países el implementar políticas de equidad de género desde el año 1990, y que luego se confirman en el año 2000 en Dákar. No obstante, ha habido pocos avances y más retrocesos en el caso peruano. Respecto al avance, podemos verlo en la Ley de igualdad de oportunidades entre hombres y mujeres, y en cuanto al retroceso, el Estado se encuentra coaccionado por grupos religiosos que rechazan cualquier avance en la igualdad entre hombres y mujeres. En las conclusiones se afirma la necesidad de implementar políticas que promuevan la equidad de género en los diferentes niveles de educación del país. Dado que, es a partir de la educación que se pueden tejer los hilos de la igualdad a nivel macro y micro, por lo que se requiere de voluntad política no solo de parte del gobierno central sino, también a nivel subnacional y de los diferentes sectores académicos.

\section{METODOLOGÍA}

Este ensayo es exploratorio y descriptivo abarca principalmente la recolección de literatura científica con el propósito de formular problemas para desarrollar una hipótesis respecto al mismo. El diseño de este estudio documental y exploratorio es no experimental y descriptivo, ya que su objetivo principal es recopilar datos e información sobre las características, aspectos o dimensiones, clasificación de las instituciones y procesos sociales (Ñaupas et al., 2014). Es necesario realizar las acciones señaladas, pues ayudan a entender el quid del problema de investigación y le sirve a la investigadora conocer cómo las variables estudiadas han evolucionado en el tiempo, así como su impacto. La autora que esta investigación pueda ser referente para futuras investigaciones con objetivos más concretos, se trabajaron con las siguientes variables para la estructura del estudio, estas son: Equidad de género, políticas educativas, género en la educación.

\section{FUNDAMENTO TEÓRICO}

\subsection{Género en la historia: derechos y ciudadanía}

Un proceso histórico busca desarrollarse a lo largo de grandes periodos que abarca aspectos estructurales como valores y creencias que luego maduran hasta convertirse en un proceso de transformación y son éstos los que sientan las bases del mundo. Dicho proceso, que ha ido evolucionado desde el feudalismo al capitalismo y de este a la industrialización y a lo largo del tiempo se fueron haciendo familiares los conceptos de crecimiento y progreso. Ello conllevó al desarrollo de diversos procesos de producción y tecnología que generó a su vez un cambio en el esquema social en cuanto a la concepción del mundo y por ende, a una nueva perspectiva de nación y Estado como superestructura. Es en ese momento, cuando el ciudadano decide ponerle fin a las relaciones de vasallaje y servidumbre a las que se encontraba sometido durante mucho tiempo, firma un nuevo pacto social que le garantice nuevos derechos (Márquez, Gutiérrez y Gómez, 2017).

No obstante, pese a los cambios sustanciales que acontecían para el hombre, las mujeres seguían siendo invisibilizadas negándoseles su condición de ciudadanas, coactando su participación en la esfera política, social y democrática. Dicha exclusión se justificaba mediante un mecanismo 
supuestamente "razonado" del proceso de naturalización de las diferencias entre mujeres y hombres. Y es así que el mundo quedó escindido en categorías binarias o dicotómicas: hombremujer; naturaleza-cultura; privado-público; objetividad-subjetividad; pasión-razón; cuerpo-mente (Márquez, Gutiérrez y Gómez, 2017; Abasolo y Montero, 2008).

Esta forma de ver la realidad hace que la población se perciba con fracturas sociales que indican diferencias en cuanto a la asunción del poder, ya sea éste económico, político o social; por lo que las desigualdades en cuanto al género se hacen más evidentes. No existe interés por parte de los y las ciudadanos/as de frenar dichas brechas, es así que la violencia simbólica se legitima y quién la recibe asume de manera directa la violencia estructural (Bourdieu, 2001). Las desigualdades se ven expresadas desde la concepción heteropatriarcal que se ha venido construyendo a lo largo de la historia y que se ha caracterizado por normalizar y promover estereotipos sexuales, generando una división en los espacios públicos y privados alimentando la exclusión, la discriminación, la inequidad y el abuso de poder que se han venido manifestando, a través de la injusticia económica, cultural y académica (Barragán, et al., 2016).

En los años setenta se cuestionan dicha concepción que era hasta ese entonces, la forma como hombres y mujeres concebían su vida y surge, principalmente en países europeos como España, Reino Unidos, Dinamarca e Italia, dos posturas para hacer frente al patriarcado: la igualdad de oportunidades y la eliminación del sexismo. La igualdad de oportunidades se explica desde el punto de vista de la justicia social que tiene como núcleo la libertad individual que se logra mediante la igualdad de acceso a la educación. Y respecto a la eliminación del sexismo, busca en la justicia social, en base a la eliminación de las barreras estructurales que aseguren respeto entre hombres y mujeres (Araya, 2001).

La propuesta que recogió América Latina fue la de igualdad de oportunidades. Sin embargo, esto no significa que se deba olvidar la situación de atraso y violencia a la que fue sometida la mujer a lo largo del tiempo. Araya afirma que un trato igualitario no garantiza la verdadera igualdad, sino que más bien perpetúa la desigualdad. Por lo que es tiempo de erradicar aquellos valores considerados tradicionalmente femeninos, y que aún las mujeres y niñas latinoamericanas siguen aplicando, por lo que es necesario el reconocimiento de las diferencias entre hombres y mujeres de un modo éticamente desafiante y políticamente transformador. Este enfoque propuesto por Araya, conduce a la equidad como política que impulsa a la educación a una dimensión ética de justicia social ofreciendo a cada quien lo que le corresponde de acuerdo con su condición personal, familiar y social (Araya Umaña, 2001). O parafraseando a Aristóteles, la igualdad debe ser para los iguales y la desigualdad para los desiguales (libro V de la Ética a Nicómaco).

\subsection{Género y Educación para cerrar brechas}

La UNESCO ha puesto en la agenda la necesidad de cerrar brechas de género en la educación, con el objetivo de que la igualdad entre hombres y mujeres sea la regla; por ello señalan la necesidad de construir sociedades más justas a través de Estados comprometidos en la igualdad de oportunidades (UNESCO, agenda 2030, ODS5). Esta visibilización de brechas de desigualdad, empezó a estudiarse con más acogida en los años setenta pues diversas autoras feministas diferenciaban al sexo del género, señalando que el género es el resultado de construcciones sociales de acuerdo con cada época y lugar y ello difería del aspecto biológico del sexo. Salgado, lo explicó como: "La categoría de género permite desarmar la naturalización de la opresión, basada en hechos biológicos (diferencia genital) que son proyectados en desigualdades sociales" (Scott, 2011; Salgado, 2006, p. 164).

Joan Scott coincide en la definición señalada por Salgado respecto al género, pero adiciona que el género es una forma primaria de relaciones significantes de poder. Asimismo, éste 
comprende cuatro elementos interrelacionados entre sí, a) el simbólico, que tiene representaciones múltiples a veces contradictorias, por ejemplo las figuras de María Magdalena y María, madre de Jesús, b) el normativo, que expresa interpretaciones de los símbolos basados en doctrinas religiosas, educativas, científicas, legales y políticas que afirman categóricamente el significado de varón y mujer, masculinas y femeninas, por ejemplo, los talleres culturales se dividen aún por actividades propias para varones (mecánica, electricidad, carpintería) y propias para mujeres (costura, cocina, primeros auxilios), c) el institucional, referido a la familia, las relaciones de parentesco, el mercado de trabajo, educación, política; por ejemplo las brechas salariales, la paridad de género en puestos de decisiones, d) el subjetivo, referido a la construcción de las identidades (Scott, 2011).

Scott, refuerza señalando que el género es un medio para identificar el abuso de poder de un sexo sobre otro dentro de la sociedad. Por lo que la aplicación del enfoque de género posibilita la medición de las brechas de inequidad social (Buquet, Cooper y Rodríguez, 2010). Asimismo, Rebollo et al. (2012) define esta perspectiva como "la defensa de la igualdad entre hombres y mujeres en el control y uso de bienes y servicios y se considera un factor clave para lograr la justicia y la cohesión social" (Rebollo et al., 2012, p. 131). De la misma forma, la Organización para la Cooperación y el Desarrollo Económicos (OCDE) (2004) afirma que el enfoque de género ayuda a leer la realidad educativa, identifica brechas y obstáculos que se producen desde la escuela; por lo que la articulación de dicha perspectiva en el entorno educativo ayuda a los y las estudiantes a visibilizar toda forma de discriminación que se produce muchas veces por el ejercicio abusivo del poder. Para las mujeres, significa también ser parte activa en los procesos de empoderamiento y cambio educativo a través de competencias y habilidades a fin de procesar formas de relación y maneras de ver el mundo (OCDE, 2004).

Para Stromquist (2002), la equidad es parte del discurso oficial pero no en cuanto a género, pues ella señala que existe un esfuerzo limitado para enfrentar las inequidades de género. Manifiesta que cuando se busca enfrentar la problemática solo se considera a las niñas pobres como las únicas afectadas en la coyuntura por lo que no se ve al género per se cómo un marcador social engarzado históricamente, que requiere un compromiso a través de todas las clases sociales de todo nivel de educación y de todas las áreas de la sociedad (Stromquist, 2002). Esta desigualdad y discriminación en la equidad afectan a la mayoría de las mujeres en el mundo quienes no se ven representadas y que según CEPAL (2017), estas formas de violencia se evidencian a través de prejuicios seculares que se encuentran estrechamente vinculados con el problema del subdesarrollo, que es el resultado principalmente de estructuras internas inadecuadas y de un sistema económico mundial profundamente injusto (CEPAL, 2017).

Desde inicios del siglo XXI la UNESCO también se ha manifestado en cuanto a brechas en la paridad de género en el sistema universitario; si bien a lo largo del S.XX estas brechas respecto al acceso a la educación se han ido reduciendo ${ }^{1}$; no obstante, tal aumento no significa que las condiciones de equidad hayan mejorado y esto porque mientras la mujer sigue aumentando peldaños académicos, su presencia se ve disminuida dentro de los espacios académicos sobre todo en postgrados, en investigación y docencia (UNESCO, 2012; Buquet Corleto et al., 2013). En México, por ejemplo, en la Universidad Nacional Autónoma de México (UNAM) las académicas representan un $42.2 \%$ de la plana docente, pero solamente un $26.9 \%$ son nombradas y de ellas, menos del $20 \%$ se desenvuelven en las áreas de investigación científica (Buquet Corleto et al., 2013).

En la Asociación Nacional de Universidades e Instituciones de Educación Superior (ANUIES), de 180 instituciones afiliadas, solo 20 son encabezadas por mujeres con cargos de directora general o rectora. En el Consejo Nacional de Educación mexicana, que se encuentra constituido por quince universidades o institutos, solo una institución se encuentra dirigida por una

\footnotetext{
${ }^{1}$ Ya no es extraño ver a mujeres estudiando una carrera profesional de diverso perfil, sin embargo, aún siguen siendo poco el avance en cuanto a acceso en algunas facultades y respecto a la permanencia.
} 
mujer (Ordorika, 2015). En Perú, solo 5 rectoras han sido elegidas desde el año 1977 hasta la actualidad $^{2}$; asimismo, se evidencias una división sexual notoria respecto a la elección de las carreras, por ejemplo, la carrera de educación ha sido mayormente elegida por las mujeres $(19,8 \%)$ que incluso duplica a la de los hombres $(9,6 \%)$. En cambio, la carrera de ingeniería tiene mayor preferencia entre los hombres; así, en el año 2017 el 30,0\% de los hombres eligieron esta carrera frente al 8,6\% en mujeres. El 7,6\% de las mujeres estudian enfermería en comparación con el 1,9\% de hombres; mientras que la carrera de psicología es estudiada por el 9,4\% de las mujeres y el 5,3\% de los hombres. Y respecto a las carreras no universitarias como las carreras técnicas, las de mayor preferencia para la población femenina están las relacionadas con las ciencias de la salud (24,2\%), educación (17,3\%), secretariado (11,4\%); las relacionadas a ciencias sociales y económicas $(10,1 \%)$, a administración de empresas $(9,1 \%)$ e informática $(8,7 \%)$. En tanto que, en la población masculina las carreras preferidas fueron las relacionadas con ingeniería $(39,6 \%)$, informática (12,5\%), educación (11,1\%) y administración de empresas $(9,0 \%)$, (Instituto Nacional de Estadìstica e Informàtica, 2018).

Además, Buquet, Cooper y Rodríguez (2010), señalan que es necesario incluir a la discusión universitaria las diferencias y diversidad en cuanto a la identidad de género, con el objetivo de erradicar los estereotipos sexistas, la violencia y la discriminación hacia las mujeres. Asimismo, introducir temas de género con perspectiva en los derechos humanos aportará a la formación de los y las estudiantes universitarios y desarrollará elementos esenciales para conocer y finalmente, acabar con las diversas formas de discriminación socialmente establecidas con base en la violencia simbólica. Estos enfoques han de formalizarse de manera preliminar en los programas de estudio, en los sistemas de evaluación y en las estructuras académicas con la finalidad de que las universidades puedan garantizar la necesidad de formar nuevos perfiles profesionales que requieren las sociedades actuales ${ }^{3}$ evidenciando de esa forma que buscan contribuir con la reducción o eliminación de brechas de desigualdad entre un género y otro. (Buquet, Cooper y Rodríguez, 2010; Bourdieu, 2001).

En el contexto de la educación básica, Alegría (2020) señala que implementar el enfoque de género tiene implicancias en la educación y en la salud pública. La autora, se sustenta en dos teorías; el primero, que alude a Foucault cuando manifiesta que la ausencia de una educación sexual reproduce discursos que conforman una "matriz patologizante de la sexualidad que tiene efectos de dominación sobre los cuerpos y la vivencia de la sexualidad", que lejos de ser una construcción de la identidad sexual de manera íntima y privada, se desarrolla de manera pública y social. Y el segundo, refiere al aspecto ideológico pues no incluir el enfoque de género en la currícula escolar, favorece discursos que promueven un sistema de opresión de la mujer y la sexualidad donde las de un nivel socioeconómico más bajo se encuentran en mayor desventaja (Alegría, 2020, p.10).

El currículo en la educación básica evidencia un sexismo explícito pues éste se refleja en lo que se enseña y en lo que no se enseña, en el lenguaje que contiene un uso regular y normativo de lo masculino para designar personas de ambos sexos y en los textos escolares, que por lo regular transmiten papeles típicamente asignados a hombres y mujeres. Ello reflejaría que la educación es usada para legitimar las desigualdades entre géneros, haciéndolas parecer como resultado de las

\footnotetext{
${ }^{2}$ La primera rectora elegida fue Ilse Wisotzki, en el año de 1977 en la Universidad de Lima. En el 2014, fue elegida en la Universidad del pacífico, Elsa del Castillo. Edith Mellado, en la Universidad Peruana Los Andes de Huancayo. María Graciela Risco de Domínguez, actual rectora en la Universidad Tecnológica del Perú y, luego de 470 años desde su fundación la Universidad Nacional Mayor de San Marcos, tuvo a su primera rectora, la Dra. Jerí Ramón Ruffner.

${ }^{3}$ En la Pontificia Universidad Católica del Perú ha venido desarrollando diplomas en "Estudios de género", Curso en "Género, Derechos y Políticas Públicas"; La Universidad Nacional Mayor de San Marcos, ha desarrollado cursos de "Estudio de género y trabajo en el Perú"; La universidad ESAN, tiene un diplomado en "Enfoque de género y políticas de igualdad". De esta forma, estas universidades contribuyen de manera significativa en la creación de una sociedad más justa y equitativa, en beneficio de la sociedad en general. Si bien se cuenta con un avance en el caso peruano, aún sigue siendo muy bajo, respecto al total de universidades licenciadas en el país.
} 
características individuales que cree tener el deber de formar individuos con características diferentes, pero lo que logra es formar ciudadanos que se inserten a una sociedad con una marcada división del trabajo. Es esa realidad la que crea la necesidad de generar individuos con una fuerza de trabajo diferenciado y jerarquizado que luego se inserta a la PEA y mantiene relaciones abusivas de poder en perjuicio del otro u otra. Esta educación mixta parte del principio de "homogenización" de la enseñanza y contribuye a la participación femenina; no obstante, el modelo masculino sigue prevaleciendo en muchos aspectos del desarrollo humano como un mandato cultural, incluso en situaciones que parecen insignificantes como la vestimenta, que muestra una evidente discriminación hacia las mujeres pues el uso de faldas impide que las estudiantes puedan desarrollar diferentes deportes o ejercicios dentro de la escuela e incluso no las ayuda a protegerse del frío (Araya, 2004; Castillo y Gamboa, 2013).

Esta diferenciación que se afirma no es evidente en el plan de clase del o la docente, tampoco se encuentra estipulado en el diseño curricular de forma taxativa, sino que se expresa a través del currículo oculto que ha venido desarrollándose a lo largo de la experiencia docente, con las actitudes, prejuicios, estereotipos y conductas machistas, que siguen repitiendo y transmitiendo como conductas arraigadas y normalizadas socialmente, en otras palabras, buscan incidir en lo que es ser hombre y lo que es ser mujer, según el establishment (Castillo y Gamboa, 2013).

Por su parte Arcos, Figueroa, Miranda y Ramos (2007), adicionalmente, distinguen cuatro tipos de currículo relacionados con el género en el ámbito educativo, y afirman que este discurso pedagógico puede presentarse de cuatro maneras: a) el formal, y en la práctica pedagógica, b) en Acción, c) Obviado y d) Oculto. El currículo formal hace referencia al enfoque pedagógico de la metodología, a los recursos, contenidos, criterios de evaluación, etc. Que normalmente suele ser consensuado por un grupo de expertos o técnicos en la educación. En el segundo, el de Acción, hace referencia a las emergencias que se generan en el espacio escolar, tales como los acontecimientos históricos, sociales o de la cultura que rodea a la institución. En el Oculto, se evidencia lo que sucede en la ejecución de los cursos, en el aula o el taller es decir, lo que no está previsto formalmente respecto a las interacciones entre docentes y estudiantes; asimismo, este juega un papel importante en la configuración de significados y valores, la mayoría de las veces no es consciente por lo que es difícil reconocerlo, por ejemplo cuando un o una docente quiere señalar la cantidad de estudiantes se expresa en masculino, obviando la existencia femenina en el aula; o incluso, introduce comentarios sexistas dentro del aula, como cuando habla de los oficios de mujeres y de hombres. Finalmente, el currículo obviado que se refiere a un conjunto de situaciones que se presentan en los procesos de enseñanza-aprendizaje, lo cuáles son los temas no tratados en clase, allí se ignoran las interrogantes respecto a temas transversales como sentimientos, necesidades, intereses, experiencias o incluso cuestiones biológicas, tales como la menstruación, la masturbación, etc. (Arcos et al., 2007).

Es por ello necesario que se incidan en políticas públicas con equidad de género, más aun en la educación básica que es un elemento necesario para la construcción de sociedades más justas y democráticas.

\subsection{Políticas Públicas para una Educación con Equidad}

La creación de una ley de equidad de género en el sistema educativo tiene como objetivo "promover y fomentar las condiciones que posibiliten la no discriminación, la igualdad de oportunidades y de igual trato entre los géneros, así también el ejercicio pleno de todos los derechos de las mujeres y su participación equitativa en la vida política". Con esta acción afirmativa se debe incluir dentro de las medidas para lograr que la equidad de género forme parte de un programa de intervención, orientada a subsanar las desigualdades preexistentes entre distintos grupos de la población. No obstante, esta política aún se sigue dilatando en todos los niveles educativos; e 
incluso, muchas veces se ve obstaculizado por grupos religiosos ${ }^{4}$ que utilizan conceptos erróneos para referirse a ésta generando mayor confusión en la población y perjudicando sobre todo a los y las que se encuentran en etapa escolar (García, 2005, p. 79).

En el caso de las instituciones de educación superior (IES), las políticas de acción afirmativa en cuanto al género debe desarrollarse en programas que fomenten la participación equitativa entre mujeres y hombres en los diferentes espacios superiores ya sea en los nombramientos, en las carreras, en áreas de conocimiento, espacios de decisión, cargos académico-administrativos, puestos o ámbitos en los que se estén expresando desigualdades en el personal académico, administrativo y en la población estudiantil con la finalidad de subsanar dicha diferenciación (Buquet Corleto, Cooper y Rodríguez, 2010). También es necesaria la promoción, la implantación, el seguimiento y la evaluación de los procesos que harán que este corpus se convierta en una práctica cotidiana en la vida nacional (García, 2005).

Por otro lado, en el ámbito escolar (educación primaria y secundaria), dichas políticas fueron discutidas en abril del año 2000 pues en ese momento, más de 1000 participantes de 164 países asistieron a Dakar (Senegal) al Foro Mundial sobre la Educación para reafirmar su adhesión a la idea de la educación como derecho humano fundamental, buscando cumplir con los objetivos de la Educación Para Todos (EPT) con el fin de señalar los objetivos que debían cumplir los países firmantes del acuerdo. Respecto a la equidad de género en dicho Marco de Acción Dakar se señaló lo siguiente:

Objetivo $5^{\circ}$ de la EPT: suprimir las disparidades entre los géneros en la enseñanza primaria y secundaria que debían cumplirse hasta el 2015 la igualdad entre los géneros en relación con la educación, en particular garantizando a las jóvenes un acceso pleno y equitativo a una educación básica de buena calidad, así como un buen rendimiento. (EPT, 2000, p. 1)

Con base en ello, la UNESCO se comprometió a realizar seguimientos periódicos a los países que asumieron el acuerdo; sin embargo, los resultados que recogieron respecto al pacto firmado fue que muchos países no lograron incluir el concepto de género en sus políticas educativas. También, se evidenció que si bien muchas mujeres han tenido acceso a la educación básica e incluso universitaria aún hace falta un mayor trabajo para que la educación resulte equitativa, por lo que en general aún falta contribuir en reducir la desigualdad que genera el acceso y la permanencia en la educación (UNESCO, 2012). En septiembre del 2000 con la finalidad de reforzar el objetivo anterior se reunieron 192 estados miembros de las Naciones Unidas en Dakar y al menos 23 organizaciones internacionales para definir una serie de Objetivos de Desarrollo del Milenio (ODM), uno de los objetivos principales fue:

Promover la igualdad de género y la potenciación de la mujer (Meta 4). Eliminar las desigualdades entre los géneros en la enseñanza primaria y secundaria, preferiblemente para el año 2005, y en todos los niveles de la enseñanza antes de fines de 2015 (UNESCO, 2002 p. 8).

Como resultado de este acuerdo se firmaron los siguientes compromiso: la Convención sobre la eliminación de todas las formas de discriminación contra la mujer (CEDAW), la

\footnotetext{
${ }^{4}$ En Perú en diciembre de 2016, se llevó a cabo el "Congreso Iberoamericano por la Vida y la Familia" y en marzo del 2017 se organizó una de las primeras marchas que tenía como lema "con mis hijos no te metas". Bajo este lema, se reunieron distintas agrupaciones religiosas, tales como: Alianza Cristiana y Misionera (ACYM), el Movimiento Misionero Mundial (MMM), Asambleas de Dios (LAD), la Comunidad Cristiana Agua viva y La Casa del Padre. La finalidad, señalar que la familia se encargaba de los hijos, en el que nadie debería entrometerse, ni siquiera los programas del Estado; de tal forma que la forma como se educa, solo debe recaer en manos de los padres, y no en la deliberación social o política (Alegría, 2020).
} 
Convención Interamericana para prevenir, sancionar y erradicar la violencia contra la mujer (Belém do Pará), la Declaración y la Plataforma de Acción de la Cuarta Conferencia Mundial sobre la Mujer (Beijing, 1995), en el Perú se aprueba la Política Nacional de Igualdad de Género con el objetivo de abordar las causas y los efectos de la discriminación estructural contra las mujeres en concordancia con las obligaciones internacionales en materia de derechos humanos, así como las políticas de Estado establecidas en el Acuerdo Nacional, el Plan Estratégico de Desarrollo Nacional y la pre-imagen del Perú al 2030 (Decreto Supremo Nº 008 -2019-MIMP).

En dicho Decreto Supremo No 008 -2019-MIMP se estipula que al 2030 y a nivel nacional, se evidenciarían los siguientes indicadores: i) la reducción a 36,8\% de la tolerancia social hacia la violencia contra las mujeres; ii) la incorporación del enfoque de género en la gestión institucional del $100 \%$ de los ministerios; iii) la reducción a 2,4\% del porcentaje de mujeres víctimas de violencia física y/o sexual en relaciones de pareja, en los últimos 12 meses; iv) el incremento de las mujeres congresistas a $40 \%$ y de las mujeres alcaldesas a $20 \%$, y v) el aumento de la tasa de paridad de los ingresos de mujeres y hombres al 86,8\% (D.S. N 008-2019 MIMP).

No obstante, la incorporación del género en los Estados participantes fue mínima, pues solo hicieron alusión a los estereotipos sexuales. Es necesario señalar que dichos acuerdos respecto a la igualdad entre hombres y mujeres no partieron con Dakar en la UNESCO; sino mucho antes, en Jomtien (1990) allí se abordó a la educación como un derecho que debía ser más equitativa y democrática en el marco de un proceso social con la presencia del Estado; no obstante, los acuerdos tampoco fueron cumplidos, y es así que se inicia en el año 1999 un nuevo diagnóstico de la educación peruana para la conferencia en Dakar. El resultado que se obtuvo fue que el Perú no realizó acciones para promover el enfoque de género en sus políticas educativas, pese a que dicha perspectiva partía de su compromiso en la Declaración mundial sobre Educación para Todos (UNESCO y TAREA, 2001; Muñoz, 2006).

Ya en Dakar, el Perú asume nuevos compromisos respecto a la reducción de brechas de desigualdad en la educación que venían siendo patentes desde décadas atrás. También se hace evidente que los "sistemas educativos y las escuelas son reflejo de la sociedad por lo que es necesario medidas para combatir la discriminación derivada de conductas y prácticas sociales, culturales y económicas" (Dakar, 2000, p. 5). Para conocer cuánto se había avanzado desde este último acuerdo, Muñoz (2006) realizó entrevistas a funcionarios del sector educación y ellos afirmaron que los acuerdos tomados fueron simplemente ignorados. Y ello se contrasta con lo recogido por la investigadora en la documentación de los años 1995 al 2001, que evidencia que los temas en cuanto a la equidad de género no fueron una prioridad en los lineamientos de las políticas del sector educación (Muñoz, 2006).

Por otro lado, en el año 2001 se promulgó la Ley $N^{\circ}$ 27558, llamada "Ley de Fomento", que hacía explícita la labor del Estado en la educación de las niñas y adolescentes rurales. Aquí, el concepto de equidad está asociado a dicha población por considerarla vulnerable: "El Estado promueve condiciones de equidad entre niños, niñas y adolescentes en áreas rurales para lo cual deben formular políticas educativas que respondan a las necesidades de ese sector, en el marco de una formación integral y de calidad para todos" (Diario oficial El Peruano, 2001, Artículo 1), pese a una intensión positiva de la normativa ésta solo evidencia ello, ser solo una intensión. No generó un impacto real en la población rural, menos en la población más vulnerable como son las menores de edad, por lo que solo siguió formando parte de la estadística de las leyes que no trascendieron dentro del país; asimismo, dicha Ley tampoco se pudo ejecutar en el área educativa. Y es así que en el periodo 2000-2005 se presenta un nuevo plan con enfoque de género donde se hacen explícitas las relaciones de poder que se detentan en la sociedad peruana para ser aplicado entre los años 2003 al 2010; sin embargo, representantes de la Iglesia católica ${ }^{5}$ se opusieron a este enfoque por

${ }^{5}$ Actualmente, muchos grupos religiosos de diversa índole eclesiástica, siguen mostrando su reticiencia a que el enfoque de género se implemente en la educación, pues consideran que son los padres o madres quienes se deben encargar de la educación sexual, 
considerarlo peligroso, y afirmaron que dicho enfoque alentaba la homosexualidad y atentaba contra los valores y las buenas costumbres de la sociedad (Muñoz, 2006).

De la misma forma Muñoz (2006), señala que recién el año 2005 el Perú publicó el Plan Nacional de Educación para Todos del Ministerio de Educación en el Foro Nacional de Educación para Todos. Por primera vez se indicaba en un plan de manera clara, la erradicación de las desigualdades de género y este tenía que ser visto como un enfoque transversal a todos los objetivos estratégicos, por lo que la equidad de género debía ser entendida como:

"Un principio de justicia que trata de eliminar todas las barreras que impiden la igualdad de oportunidades económicas, políticas, de acceso a la educación, a los recursos y a los servicios básicos entre hombres y mujeres, por lo que equidad de género connota la igualdad de derechos de todos y todas" (Plan Nacional de Educación para Todos, 2005, p. 80).

Sin embargo, desde el año 2002 hasta el día de hoy existe una "lenta" y "poco articulada" incorporación de los temas de género en el Perú. La implementación del Currículo Nacional de la Educación Básica (CNEB) marca un "hito" según Muñoz, además, que tiene sus idas y retrocesos. Estos atrasos, causados por los grupos religiosos que niegan cualquier desarrollo científico en cuanto al género. La incorporación del enfoque de género ha mostrado un menor nivel de avance en comparación con otros sectores pese a que este concepto se ha ido introduciendo desde hace tres décadas hasta ubicarse como uno de los siete enfoques ${ }^{6}$ transversales del Currículo Nacional de la Educación Básica que se implementa desde el año 2003 de acuerdo con la Ley de Educación.

\section{DISCUSIÓN}

Para García (2005), "la escuela es a través del proceso de socialización, la generadora de prácticas discriminatorias" (García, 2005, p. 71). Sobre este punto, Fuentes (2016) concuerda y señala que, para acabar con dichas prácticas es importante desarrollar una educación multicultural con adopción de los lentes de género, ya que esto permitirá examinar y comprender de manera distinta la construcción del conocimiento y su contexto histórico, ético, político y social, entre otros asuntos. Incluir este enfoque desde el diseño curricular dará las herramientas para establecer desigualdades y exclusiones en la construcción, difusión y apropiación del saber (Fuentes, 2016). Por lo tanto, "la solución consiste en re-trabajar el actual sistema escolar para modificarlo sin conflictos" (García, 2005, p. 71).

Asimismo, Palomar (2005), García (2005) y Stromquist (2002) muestran que el mundo universitario no está exento de las desigualdades de género. Se han documentado diferencias en todos los niveles de la educación básica y en todos los planos de la vida de las universidades: desde las desigualdades numéricas en la ocupación de puestos docentes, en las tomas de decisiones y en la investigación, donde suelen encontrarse siempre más varones que mujeres (Palomar, 2005). Del mismo modo, García señala que dichas estructuras de poder vienen siendo recogidas de antaño y refuerza la idea de que la escuela es solo un espacio de transmisión del conocimiento masculino, dado que el sistema educativo solo presenta el punto de vista de lo que constituye la experiencia masculina (García, 2005).

\footnotetext{
reproductiva de sus hijos, es decir desde el ámbito privado. Alegría (2020) señala que es son discursos patologizante y de dominación que afectan gravemente a quienes pertenecen a los niveles socioeconómicos más bajos. Alegría también señala que los acuerdos arribados en Dakar, suceden en el gobierno de Alberto Fujimori, es así que el Estado se compromete, de manera general, a incentivar políticas para las mujeres, lo que permitió intervenir mediante programas de "planificación familiar". Contrariamente, lo que se presentó como una manera de empoderar a las mujeres sobre su vida sexual, condujo a la aplicación de un conjunto de políticas de esterilización que se implementaron privilegiando la maximización del capital sobre los derechos de los cuerpos de las mujeres (p.14).

${ }^{6}$ Los enfoques transversales son, entre otros: 1 . enfoque de derechos, 2. enfoque inclusivo de atención a la diversidad, 3. enfoque intercultural, 4. enfoque de igualdad de género, 5. enfoque ambiental, 6) enfoque orientado al bien común, 7) enfoque búsqueda de la excelencia.
} 
En cuanto a los estudios con enfoque de género en las universidades de educación superior, Palomar reconoce su legitimidad y valor, pues estas tienen efectos sobre la calidad misma de la investigación científica en este campo (Palomar, 2005). En la misma línea, Bolaños añade que los diseños curriculares de pregrado y posgrado deben contemplar dos momentos decisivos: "[...] la construcción de un marco socio histórico y de un marco epistemológico de la disciplina", y que en esta etapa se deben incorporar los enfoques multiculturales e inclusivos (Bolaños, 2003, p.21). En la misma tesitura, García (2005) señala que la "universidad como espacio vital en la formación de profesionales para el trabajo y la sociedad debe también generar una cultura de la información por sexo" (p. 86). El objetivo de este enfoque es el de "promover y fomentar las condiciones que posibiliten la no discriminación, la igualdad de oportunidades entre los géneros; el ejercicio pleno de los derechos de las mujeres y su participación equitativa en la vida política, cultural, económica y social del país" (p. 79). Su ejecución busca desarrollar políticas públicas con una perspectiva de género en la educación en todos los niveles y la puesta en marcha de programas y acciones coordinadas o conjuntas (García, 2005, pp. 70-87).

\section{CONCLUSIÓN}

La historia ha revelado que, si bien ha habido cambios sustanciales en el ámbito educativo, aquí solo se benefició un género, el masculino; la mujer, por otro lado, sigue siendo discriminada por diversas razones: políticas, sociales, culturales. No obstante, pese a tener muchos obstáculos en diversos espacios sociales, ella se ha ido posicionando en el plano educativo desde los niveles básicos y ha buscado mantenerse así, obviamente esto no ha sido fácil pues se vuelve más complicado mantenerse en la universidad, ya que debe luchar contra las diferentes brechas de desigualdad que existen, desde el acceso y la permanencia por señalar algunos ejemplos, más aún cuando la carrera que eligen es predominantemente masculina. Según la UNESCO a diferencia de los hombres, las mujeres buscan concluir o permanecer en el ámbito académico y conforme van subiendo peldaños la presencia femenina disminuía en las maestrías y doctorados; esto es una clara señal de falta o nula formulación de políticas inclusivas con énfasis en género, así como su institucionalización. No hay un apoyo del mundo académico o político respecto al avance académico de la mujer, pues muchas de ellas deben cortar su avance con la maternidad o cuidado de personas mayores como sus padres y su retorno suele ser muy complicado. Esta problemática no la sufren los hombres en la misma magnitud, por lo que ellos pueden seguir creciendo sin problemas por lo que es necesario un replanteo de las políticas, sobre todo en las diversas comunidades científicas, académicas y organizacionales que conforman la Universidad.

Es cierto que las mujeres afrontan mayores desventajas en las instituciones de educación básica y superior y diversos estudios constatan la enorme desigualdad en la educación terciaria, donde hay escasa o nula participación femenina en niveles directivos, segregación horizontal según campos del conocimiento, currículos masculinizados y discriminación y violencia sexual. Pese a que las evidencias son abrumadoras se ha hecho poco para cambiar esta situación, pues aún se siguen tratando a los y las estudiantes en términos de género masculino, la bibliografía en un silabo sigue siendo predominantemente de autores hombres o las propuestas de lectura sugeridas al alumnado/a es de autores masculinos, entre otros. Si las autoridades académicas y políticas no hacen algo al respecto, las brechas y la inequidad seguirán siendo la regla, vulnerando derechos como la no discriminación, derecho a la igualdad en todas sus formas y el acceso a una educación libre de violencia.

Este ensayo ha rescatado las siguientes preguntas con base en la revisión de la literatura científica ¿Cuál es la importancia del género en la educación?, ¿De qué modo el ámbito escolar sigue promoviendo brechas de género?, ¿Cómo lograr una educación más inclusiva y con equidad de género? ¿Cómo lograr la igualdad de género en la educación? ¿Por qué es importante la perspectiva de género? Algunos desafíos entre ellos está profundizar y formalizar la política 
institucional de promoción de equidad; diseñar un protocolo institucional de atención a situaciones de violencia de género, acoso y cualquier forma de discriminación dentro de espacios académicos, visibilizar la importancia de transversalizar la igualdad de género. Dichas investigaciones mayormente están desarrolladas con un enfoque cualitativo - analítico a partir de una teoría social y política. La investigadora, en base dicha revisión pudo formular el siguiente problema ¿Existe una educación inclusiva y con equidad de género en la política educativa peruana? si bien existen reformas educativas con enfoque de género que se han plasmado en normativas, agendas, planes nacionales entre otros, estas no han tenido un resultado satisfactorio a lo largo del tiempo. Este problema, debe aterrizar en acciones que se sustenten en la implementación de programas académicos y técnicos con equidad de género que puedan colaborar a disminuir las desigualdades entre el hombre y la mujer en la educación de los diferentes niveles.

¿Qué se debe hacer? Es conveniente que quienes manejan los ministerios de manera transversal inserten el enfoque de género en las políticas públicas, también se debe destinar un presupuesto sensible al género para la implementación de programas que incidan en la reducción de brechas. Hasta el momento se ha dejado solo al Ministerio de la Mujer que se encargue de transversalizar el género como único responsable, como si esto fuese solo responsabilidad de un género o de un ministerio. Es obligatoria la participación de todas las carteras en colaboración con las universidades para erradicar esta forma de discriminación, el presupuesto en necesario, dado que sin dinero lo único que se espera es buena voluntad de los actores/as, más no un efecto real que impacte en la sociedad. Desde Jomtien en 1990 se sigue la política de la buena voluntad, más no con actuaciones efectivas y directas. Es imperativo que el país asuma el compromiso dos veces realizado en Dakar.

\subsection{Limitaciones del estudio}

Una de las mayores limitaciones del estudio es que existe muy poca investigación de corte cuantitativo o aplicada por lo que no se encontró literatura que evidencie el impacto de insertar la equidad de género en las políticas educativas. Si bien ya la equidad de género se encuentra plasmada en ODS5 como meta a cumplirse, en muchos países de la región la inserción de este objetivo recién se encuentra de forma incipiente.

No obstante, se pudieron encontrar marcos conceptuales, respecto a los conceptos: equidad, género, desigualdad, paridad, perspectiva de género, discriminación, violencia, trasversalización, paridad. Se tomó como referente los antecedentes de instrumentos internacionales en materia de derechos humanos, organismos internaciones, tales como UNESCO, BID, OCDE; CEPAL, OIT, ONU también fueron referentes para el trabajo autores como: Bourdieu, Legarde, Stromquist, Ordorika, Araya y Muñoz. Asimismo, los y las autoras citadas están de acuerdo que existen brechas de género en el sector educativo y que se sustentan en desigualdades estructurales, y que se siguen manteniendo hasta la actualidad, pese a que muchos países han evidenciado que ello es un problema que discrimina y quita oportunidades a las mujeres a lo largo de su desarrollo. Es conveniente señalar, que en los países que se vienen aplicando políticas educativas con perspectiva de género han logrado un avance en la reducción de brechas, sin embargo, el camino es largo, y éste enfoque debe ser aplicado de manera longitudinal, por lo que los resultados se podrán apreciar en los siguientes años. También es necesaria la aplicación del enfoque de género en la política educativa, de cara al cumplimiento de las metas de los ODS, ya que se requiere de una intervención directa desde la raíz de la desigualdad de género, trabajarlo en la sociedad y en la escuela.

\section{REFERENCIAS}

Abasolo, O. (2008). Guía didáctica de ciudadanía con perspectiva de género. Igualdad en la diversidad para el profesorado de segunda etapa ESO y Bachillerato. Obtenido de Instituto de la Mujer: 
http://www.inmujer.gob.es/en/areasTematicas/educacion/publicaciones/docs/guia_didactica_ciudadania_FUHE M.pdf.

Alegría, A. (2020). La controversia en torno al enfoque de género en el currículo nacional de educación básica en el Perú por parte de la campaña \#ConMisHijosNoTeMetas. La Colmena, (13), 8-17. Recuperado a partir de https://revistas.pucp.edu.pe/index.php/lacolmena/article/view/23438Araya, S. (2001). La equidad de género en la educación. Revista de estudios de género. La ventana Vol. 2 Núm. 13 Pág. 159-187 Recogido de https://dialnet.unirioja.es/metricas/documentos/ARTREV/5202171

Araya, S. (2004). Hacia una educación no sexista. Actualidades Investigativas en Educación, vol. 4, núm. 2, pp. 1-13. DOI: https://doi.org/10.15517/aie.v4i2.9088

Arcos, E., Figueroa, V., Miranda, C., y Ramos, C. (2007). Estado del arte fundamentos para la construcción de indicadores de género en educación. Estudios Pedagógicos,vol. 33, núm 2 pp. 121-130. DOI: https://doi.org/10.4067/S0718-07052007000200007

Barragán, F., Pérez-Jorge, D., y Marrero-Morales, S. y.-F. (2016). Gender Violence and Prisoners: Action Research and Pedagogy. The new educational review,vol. 43, pp. 125-135. DOI: https://doi.org/10.15804/tner.2016.43.1.10

Bolaños, Carolina, (2003). Curriculum universitario género sensitivo e inclusivo. Revista de Ciencias Sociales, Vol. 4, núm. 102, Universidad de Costa Rica, pp. 71-78 DOI: https://doi.org/10.15517/aie.v5i4.9181

Bourdieu, P. (2001). Fundamentos de una teoría de la violencia simbólica. Barcelona: Popular.

Buquet Corleto, A., A. Cooper, J., y Rodríguez Loredo, H. (2010). Sistema de indicadores para la equidad de género en instituciones de educación superior. México: Universidad Autónoma de México. Recogido: https://cieg.unam.mx/img/igualdad/eisistindi.pdf

Castillo Sánchez, M., y Gamboa araya, R. (2013). La vinculación de la educación y género. Actividades Investigativas en Educación, vol. 13 núm. 1, pp. 113 -133. DOI https://doi.org/10.15517/AIE.V13I1.11719

Comisión Económica para América Latina y el Caribe - CEPAL (2017). 40 años de Agenda Regional de Género. Conferencia Regional sobre la Mujer de América Latina y el caribe. Santiago: Naciones Unidad.

Fuentes Vásquez, Lya (2016). ¿Por qué se requieren políticas de equidad de género en la educación superior?, Revista Nómadas, vol. 44, pp. 65-83. DOI: https://doi.org/10.30578/nomadas.n44a4

García Guevara, P. (2005). Género, Educación y Política Pública. La Ventana, núm. 21, pp. 70-89. Recuperado de https://www.redalyc.org/pdf/884/88402103.pdf

González Jiménez, R. M. (2003). Diferencias de género en el desempeño matemático de estudiantes de secundaria. Educación Matemática, pp. 129-161. Recogido de https://www.redalyc.org/pdf/405/40515206.pdf

Instituto Nacional de Estadìstica e Informàtica. (20 de junio de 2018). Encuesta Nacional de Hogares sobre condiciones de vida y pobreza, 2017. Obtenido de INEI: webinei.inei.gob.pe

Márquez, Y., Gutiérrez, J., y Gómez-Galdona, M. (2017). Equidad, Género y Diversidad en Educación. European Scientific Journal, pp. 135-158. DOI: https://doi.org/10.19044/esj.2017.v13n7p300

Muñoz, F. (2017). Las políticas educativas y la incorporación de género en la educación (1990-2016): un campo en disputa. Tarea, 94, 14-24

Muñoz Cabrejo, Fanny (2006). Caminos Cruzados: Género en las Políticas Educativas en el Perú en los últimos diez años. Educación y Sociedad Campinas, 385-406.

OCDE (2004). Informe del comité técnico sobre el sistema educativo chileno. MINEDUC, 20-29.

Ordorika, I. (2015). Equidad de género en la Educación Superior. Revista de la Educación Superior, pp. 7-17. DOI: https://doi.org/10.1016/j.resu.2015.06.001

Palomar Verea, Cristina (2005). La política de Género en la Educación Superior. Revista la Ventana, núm. 25, pp. 7-43. DOI: https://doi.org/10.32870/lv.v3i21.739

Rebollo, M., Piedra, J., Sala, A., Assumpta, C., Saavedra, J., y Bascón, M. (2012). La equidad de género en educación: análisis y descripción de buenas prácticas educativas . Revista de Educación IdUs, pp. 129-152. DOI: https://doi.org/10-4438/1988-592X-RE-2012-358-186

Scott, Joan (2011). El género, todavía una categoría útil para el análisis histórico. En La Manzana de la Discordia, enero-junio, vol. 6, número 1, pp. 95-101.

Stromquist, N. P. (2002). La equidad de género y las reformas educativas en el contexto del neoliberalismo. Debates en Sociología, núm. 27, pp. 71-88.

UNESCO (2012). World Atlas of Gender Equality in Education. Paris: UNESCO.

Valls, R. (2008). Violencia de género en las universidades españolas. Memoria 2006-2008. Barcelona: (Exp. 50/05). Recogido

de:

https://www.inmujeres.gob.es/areasTematicas/estudios/estudioslinea2009/docs/768Violgenerouniv.pdf 